\title{
Searches for Resonances Decaying to Top
}

\author{
Jörg Meyer \\ Göttingen University - II. Physikalisches Institut \\ Friedrich-Hund-Platz 1, 37077 Göttingen - Germany
}

\begin{abstract}
Searches for resonances decaying to top pairs in $p \bar{p}$ collisions at $\sqrt{s}=1.96 \mathrm{TeV}$ are presented. An upper limit on the production of a narrow width resonance is given using $2.1 \mathrm{fb}^{-1}$ data collected by the D0 experiment. Limits on the couplings of a massive gluon are given and a measurement of the differential cross section $d \sigma / d M_{t \bar{t}}$ is presented using $1.9 \mathrm{fb}^{-1}$ data collected by the CDF experiment.
\end{abstract}

\section{Introduction}

The top quark was discovered in 1995 by the CDF and D0 collaborations [2, 3]. During Run I (1992-1996) both experiments collected a data set of about $125 \mathrm{pb}^{-1}$. In 2001 Run II has started operating with a center-of-mass-energy of $\sqrt{s}=1.96 \mathrm{TeV}$ and an increased luminosity. One of the main aims is to further investigate the properties of top quarks. End of 2006 there was first evidence for single top production [4]. At present, the top quark mass is measured with a precision of $0.8 \%$ [5] and the $t \bar{t}$ production cross section is measured with a precision of about $11 \%$ [6]. CDF and D0 have recorded more than $3 \mathrm{fb}^{-1}$ of data. The increased size of top quark samples allows to precisely study the production mechanism of top quarks and to search for physics beyond the standard model (SM).

In the SM there is no resonant production of $t \bar{t}$ pairs. The only exception might be a heavy higgs boson decaying to $t \bar{t}$. However, many models beyond the SM suggest yet unknown resonances. There are $Z$-like bosons in extended gauge theories [7], Kaluza Klein excitations of the gluon or $Z$ boson [8, 9], axigluons [10], resonances in topcolor models [11], and others. Top quarks almost exclusively decay into to $W$ boson and a $b$ quark. The final state of a $t \bar{t}$ event is determined by the decay modes of the to $W$ bosons. The presented searches are performed in the lepton + jets channel $t \bar{t} \rightarrow W^{+} W^{-} b \bar{b} \rightarrow l \overline{\nu_{l}} q \overline{q^{\prime}} b \bar{b}$. In the final state there is one isolated high $p_{T}$ lepton $(l=e, \mu), \geq 4$ jets, and missing $E_{T}$. Both experiments apply $b$-tagging to improve the signal-to-background ratio. The main background is the SM $t \bar{t}$ production.

In the following a search for a narrow width resonance by the D0 experiment [12], and a search for a massive gluon [13] and a measurement of the differential cross section $d \sigma / d M_{t \bar{t}}[14]$ by the CDF experiment is presented.

\section{Reconstruction of the $t \bar{t}$ Invariant Mass Distribution}

All three analyses need to reconstruct the $t \bar{t}$ invariant mass distribution $M_{t \bar{t}}$.

D0 considers 3 jet events and $\geq 4$ jet events. $M_{t \bar{t}}$ is calculated from the 4-momenta of the jets, the charged lepton, and the neutrino. The neutrino $p_{T}$ is measured as missing $E_{T}$, and the longitudinal momentum is obtained by solving $M_{W}^{2}=\left(p^{l}+p^{\nu}\right)^{2}$. If there are two solutions the one with the smallest $\left|p_{z}^{\nu}\right|$ is taken.

CDF only considers events with exactly 4 jets. $M_{t \bar{t}}$ is reconstructed using the Dynamical Likelihood Method $[15,16,17]$. The production matrix element is not used to avoid any

DIS 2008 
bias by assuming a certain production mechanism.

The measurement of $d \sigma / d M_{t \bar{t}}$ is performed using events with $\geq 4$ jets. $M_{t \bar{t}}$ is calculated from the 4-momenta of the 4 leading jets, the charged lepton, and the missing $E_{T}$.

\section{Search for Narrow Resonance}

D0 performed a generic search for a narrow resonance $X \rightarrow t \bar{t}$. The width $\Gamma$ is assumed to be small compared to the detector mass resolution. A data set of $2.1 \mathrm{fb}^{-1}$ was analyzed. 731 events were selected in the $e+$ jets channel, and 565 events in the $\mu+$ jets channel. The total expected background is $692 \pm 26$ and $524 \pm 23$ events, respectively. There is no significant deviation from the SM expectation for the reconstructed $M_{t \bar{t}}$ distribution. The hypothesis of a narrow resonance is tested using a Bayesian approach. The result is an upper limit on the production cross section $\sigma_{X} \times B(X \rightarrow t \bar{t})$ as a function of the resonance mass $M_{X}$. The expected and observed limit at 95\% C.L. is shown in Fig. 1. This generic search can be interpreted in the context of a topcolorassisted technicolor model. It predicts the existence of a leptophobic $Z^{\prime}$, i.e. a massive

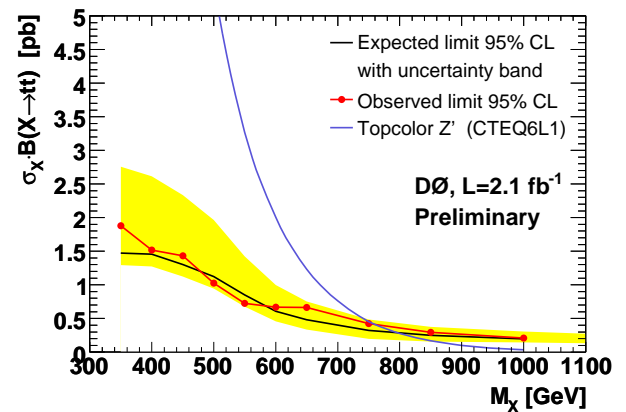

Figure 1: Expected and observed 95\% C.L. upper limits on $\sigma_{X} \cdot B(X \rightarrow t \bar{t})$ compared with the predicted top-color-assisted technicolor cross-section for a $Z^{\prime}$ boson with a width of $\Gamma_{Z^{\prime}}=0.012 M_{Z^{\prime}}$ as a function of the resonance mass $M_{X}$. The shaded band gives the $\pm 1 \sigma$ uncertainty in the SM expected limit.

Z-like boson that does not couple to leptons,

weakly and symmetrically couples to the first two quark generations, and strongly couples to the third generation. The predicted topcolor $Z^{\prime}$ production and the measured limit for a narrow resonance lead to an exclusion of a $Z^{\prime}$ at $95 \%$ C.L. with a mass of $M_{Z^{\prime}}<760 \mathrm{GeV}$ and a width of $\Gamma_{Z^{\prime}}=0.012 M_{Z^{\prime}}$.

\section{Search for Massive Gluon}

In contrast to a $Z^{\prime}$ boson a massive gluon is a color octet particle. CDF sets limits on the coupling strength $\lambda$ of such a massive gluon $G$ as a function of the gluon mass $M_{G}$ and the gluon width $\Gamma_{G}$. The SM s-channel top production $q \bar{q} \rightarrow g \rightarrow t \bar{t}$ and the production via a massive gluon $q \bar{q} \rightarrow G \rightarrow t \bar{t}$ interfere and therefore cannot be separated. The SM $t \bar{t}$ production is also treated as signal. A data set of $1.9 \mathrm{fb}^{-1}$ was analyzed. The coupling strength was extracted with an unbinned likelihood fit. The fit was performed for $\Gamma_{G} / M_{G}=$ $0.05,0.10,0.20,0.30,0.40$, and 0.50. All fitted coupling strength are consistent with the SM prediction within $\approx 1.7 \sigma$. The obtained expected and observed limits for $\Gamma_{G} / M_{G}=0.05$ and $\Gamma_{G} / M_{G}=0.5$ are shown in Fig. 2. 

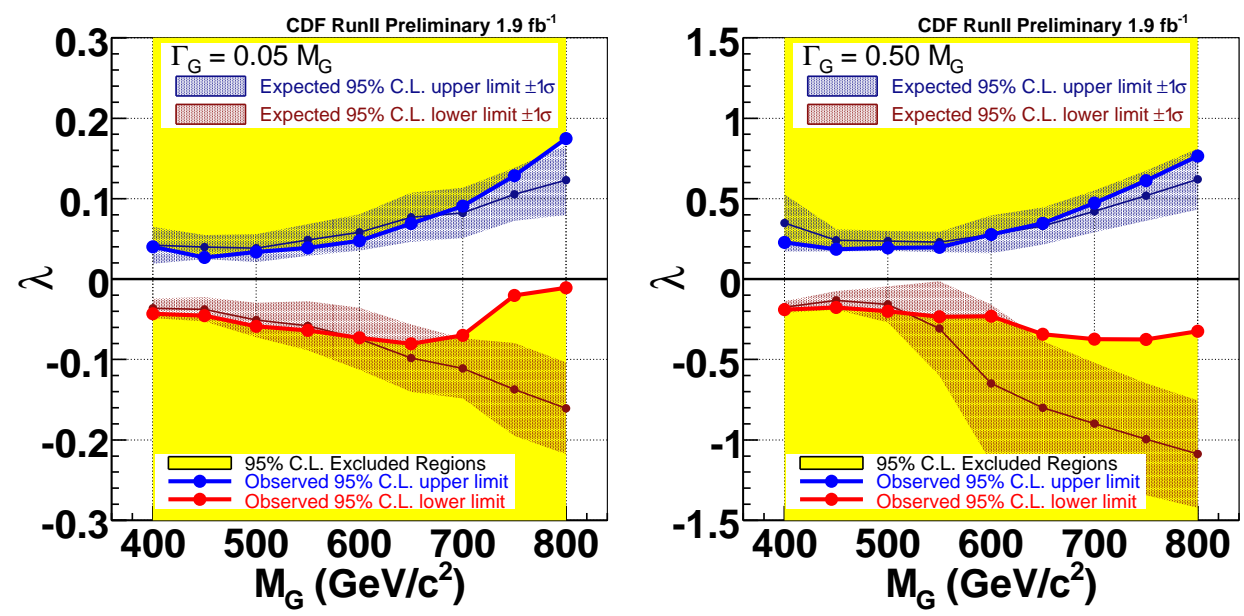

Figure 2: Limits on the coupling strength as a function of the gluon mass for $\Gamma / M=0.05$ (left) and $\Gamma / M=0.5$ (right).

\section{$5 \quad$ Measurement of $d \sigma / d M_{t \bar{t}}$}

The measurement of $d \sigma / d M_{t \bar{t}}$ is a generic test of the SM top production. The cross section is defined by

$$
\frac{d \sigma^{i}}{d M_{t \bar{t}}}=\frac{N_{i}-N_{i}^{\mathrm{bkg}}}{\mathcal{A}_{i} \int \mathcal{L} \Delta_{M_{t \bar{t}}}^{i}},
$$

where $N_{i}$ is the number of events, $N_{i}^{\text {bkg }}$ is the number of background events, $\mathcal{A}_{i}$ is the acceptance in the bin $i, \int \mathcal{L}$ is the integrated luminosity, and $\Delta_{M_{t \bar{t}}}^{i}$ is the width of the mass bin $i$. The reconstructed $M_{t \bar{t}}$ distribution is distorted from the true distribution by several effects like object resolutions and acceptance. To correct for these effects

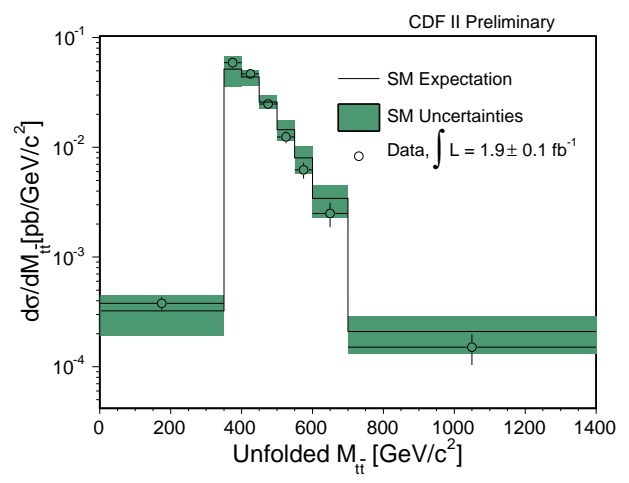

Figure 3: $d \sigma / d M_{t \bar{t}}$ in $1.9 \mathrm{fb}^{-1}$ of $W+\geq 4$ jets data. a Singular Value Decomposition unfolding technique is applied [18]. This is a linear transformation of $M_{t \bar{t}}$ bins. The transformation matrix is obtained from the Monte Carlo simulation. In Fig. 3 the measured differential cross section $d \sigma / d M_{t \bar{t}}$ is shown as a function of the unfolded $M_{t \bar{t}}$. The SM expectation for a $t \bar{t}$ cross section of $7.8 \mathrm{pb}$ is also given. There is no significant deviation from the SM. The consistency of both distributions is tested by pseudo-experiments leading to an observed $p$-value of 0.45 .

DIS 2008 


\section{References}

[1] Slides: http://indico.cern.ch/contributionDisplay.py?contribId=119\&sessionId=15\&confId=24657.

[2] F. Abe et al. Observation of Top Quark Production in $p \bar{p}$ Collisions with the Collider Detector at Fermilab. Phys. Rev. Lett., 74:2626-2631, 1995.

[3] S. Abachi et al. Observation of the Top Quark. Phys. Rev. Lett., 74:2632-2637, 1995.

[4] V. M. Abazov et al. Evidence for production of single top quarks and first direct measurement of $|\mathrm{Vtb}|$. Phys. Rev. Lett., 98:181802, 2007.

[5] Tevatron Electroweak Working Group, for the CDF, and D0 collaborations. A combination of CDF and D0 results on the mass of the top quark, 2008.

[6] V. M. Abazov et al. Measurement of the $t \bar{t}$ production cross section in $p \bar{p}$ collisions at sqrt(s) $=1.96$ tev. Physical Review Letters, 100(19):192004, 2008.

[7] A. Leike. The phenomenology of extra neutral gauge bosons. Physics Reports, 317:143, 1999.

[8] Thomas G. Rizzo. Testing the nature of kaluza-klein excitations at future lepton colliders. Phys. Rev. $D, 61(5): 055005$, Feb 2000.

[9] Ben Lillie, Lisa Randall, and Lian-Tao Wang. The bulk rs kk-gluon at the lhc. Journal of High Energy Physics, 2007(09):074, 2007.

[10] L. M. Sehgal and M. Wanninger. Forward - backward asymmetry in two jet events: Signature of axigluons in proton - anti-proton collisions. Phys. Lett., B200:211, 1988.

[11] Christopher T. Hill and Stephen J. Parke. Top quark production: Sensitivity to new physics. Phys. Rev. D, 49(9):4454-4462, May 1994.

[12] The D0 collaboration. Search for $t \bar{t}$ resonances in the lepton + jets final state in $p \bar{p}$ collisions at $\sqrt{s}=1.96$ TeV, 2008. http://www-d0.fnal.gov/Run2Physics/WWW/results/prelim/TOP/T65/T65.pdf.

[13] The CDF collaboration. A search for massive gluon decaying to top pair in lepton+jet channel, 2008. http://www-cdf.fnal.gov/physics/new/top/confNotes/cdf9164_MG_DLM.pdf.

[14] The CDF collaboration. Measurement of the $t \bar{t}$ differential cross section, $d \sigma / d m_{t \bar{t}}, 2008$. http://wwwcdf.fnal.gov/physics/new/top/confNotes/cdf9157_dsigma_dmtt_pub.ps.

[15] Kunitaka Kondo. Dynamical likelihood method for reconstruction of events with missing momentum. i. method and toy models. J. Phys. Soc. Jpn., 57:4126, 1988.

[16] Kunitaka Kondo. Dynamical likelihood method for reconstruction of quantum process, 2005.

[17] A. Abulencia et al. Measurement of the top quark mass with the dynamical likelihood method using lepton plus jets events with b-tags in $p \bar{p}$ collisions at sqrt(s) $=1.96 \mathrm{TeV}$. Physical Review D (Particles and Fields), 73(9):092002, 2006.

[18] Andreas Hocker and Vakhtang Kartvelishvili. SVD Approach to Data Unfolding. Nucl. Instrum. Meth., A372:469-481, 1996. 